\title{
Web review: A few assorted websites in radiology
}

\author{
IK Indrajit \\ Department of Radiodiagnosis \& Imaging, Command Hospital (Air Force), Bangalore - 560 007, Karnataka, India \\ Correspondence: Dr. IK Indrajit, Department of Radiodiagnosis \& Imaging, Command Hospital (Air Force), Bangalore - 560 007, Karnataka, \\ India.E-mail: inji63@gmail.com
}

A few useful websites dealing with assorted topics in radiology, which are available at present on the Internet, are reviewed below.

1. Gamuts in radiology at http://rfs.acr.org/gamuts/data/ GamutsTC.htm is the web version of the $4^{\text {th }}$ edition of the textbook, Reeder and Felson's Gamuts in Radiology. It covers many modalities of imaging, including USG, CT scan, MRI, mammography, angiography, and plain films. The current version of this textbook has more than 1700 lists of differential diagnoses covering regions like skull and brain, head and neck, spine, cardiovascular, chest, etc., and subjects like topical diseases. There are special sections on MRI of the central nervous system and body and obstetrical USG, with over 7500 teaching images.

2. Manual on contrast media from the American College of Radiology is available at http://www. acr.org/SecondaryMainMenuCategories/quality_ safety/contrast_manual/FullManual.aspx. There are several chapters dealing with a wide range of topics, including patient selection and preparation strategies, adverse effects of iodinated contrast media, contrast nephrotoxicity, contrast media in children, iodinated gastrointestinal contrast media, nephrogenic systemic fibrosis, treatment of contrast reactions, etc. Educative tables are also available concerning the indications for the use of iodinated contrast media, categories of reactions, an ABCD approach for patient evaluation and treatment, management of acute reactions in adults, and equipment for emergency carts.

3. Radiography is an educational portal from SUNY Upstate Medical University, Syracuse, NY and available at http://www.upstate.edu/radiology/ rsna/radiography/. It has several sections covering techniques, X-ray tube voltage $(\mathrm{kV})$, current-exposure time product (mAs), resolution, focal spot pixel size, scatter and its relationship with collimation, $\mathrm{kV}$, and dual-energy radiography. Special sections on exposure and image artifacts are also available. A feature on radiographic artifacts is on offer at http://www.upstate. edu/radiology/rsna/radiography/artifact/.

4. E-radiology at http://eradiology.bidmc.harvard.edu/ is authored by Gillian Lieberman, from the Department of Radiology, Beth Israel Deaconess Medical Center. There are educative sections titled Interactive Tutorials in Radiology, Classics Collection, Learning Lab, and Living Anatomy. Sections covering assessment of abdominal pain, chest pain, low back pain, hematuria, breast imaging, diabetes, and AIDS are also available. On offer is a dedicated section on MRI Atlas of the Abdomen and Chest Anatomy for first-year students. Classics collection is 'a compendium of images demonstrating the classic radiological findings of a multitude of abnormalities.'

5. Radiology Masterclass, sourced at http:// radiologymasterclass.co.uk/index.html, is 'a growing resource in medical imaging teaching. There are educative tutorials dealing with chest $X$-ray, abdomen $\mathrm{X}$-ray, general $\mathrm{X}$-ray tutorials, basics of X-ray physics, objective structured clinical examination (OSCE) presentation tips, intravenous urogram (IVU) guide, common fractures, common orthopedic conditions, etc.

6. Clinical Practice Guidelines at http://www.sirweb.org/ clinical/all.shtml is a quality improvement initiative offered by the Society of Interventional Radiology (SIR) and deals with safety guidelines, consensus, policy and position and standards. The documents that facilitate quality improvement make up a long list and include guidelines for establishing a quality improvement program in interventional radiology, for percutaneous needle biopsy, for central venous access, for transcatheter embolization, for percutaneous transhepatic cholangiography, for biliary drainage and percutaneous cholecystostomy, for transjugular intrahepatic portosystemic shunt (TIPS), for reporting and archiving of interventional radiology procedures, 
for percutaneous management of acute limb ischemia, and for reducing radiation risks for patients and staff. There is also a clinical expert consensus document on carotid stenting.

7. Ultrasoundpedia at http://www.ultrasoundpaedia. com/index.html is 'a portal to a world of ultrasound education, and is intended for sonographers, sonologists, and practitioners.' The site 'provides upto-date ultrasound education and links to quality useful sites.' Illustrative sections on upper abdomen, urology, gynecology, obstetrics, musculoskeletal system, vascular system, and small parts are available here.

8. MDCT at http://www.mdct.net/mdct/eng/index. html has different sections titled MDCT Technology, Technical Considerations, Contrast Media (CM) Safety, and Continuing Medical Education (CME) video training, and Newsletter. There are sections with educative topics like CT colonography, CT perfusion of the liver, and novel perfusion $\mathrm{CT}$ techniques in neurovascular imaging, to name a few. Also provided is a Workstation Face-Off at the International Symposium on Multidetector-Row CT. Few tools like dose-flux calculator and GFR calculator are also provided.

9. eMRI at http://www.imaios.com/en/e-Courses/e-MRI/ is a step-by-step, free, interactive course on MRI, supported by IMAIOS. IMAIOS is a medical website offering different e-learning tools for healthcare professionals; the site covers topics like nuclear magnetic resonance, instrumentation and safety, MRI signal, spatial encoding, image formation, sequences, fat, water and contrast agents, image quality and artifacts, parallel imaging, MRI angiography, cardiac MRI, perfusion imaging, diffusion-weighted MRI and DTI, functional MRI, and MRI spectroscopy. A special section on 3T/ultra-high-field MRI is available. Also available from IMAIOS are neatly compiled sections titled e-Anatomy, Clinical Cases, Virtual Workshops, and Exam Bank.

10. ReviseMRI.com at http://www.revisemri.com/ is a revision aid presented in an educational format. Its contents are varied and comprise sections like questions and answers, web-based animated tutorials, interactive learning tools, and links. The interactive questions and answers currently available are related to basic physics of MRI, creating an MRI image, MRI safety, pulse sequences, K-space, image artifacts, and equipment and quality assurance.

\section{End Piece}

The web portal focusing on the Preconception and Prenatal Diagnostic Techniques Act (PC-PNDT Act) 1994 is on offer at http://pndt.gov.in/. At this site many sections can be browsed, one of which links to 'The Handbook on Preconception and Prenatal Diagnostic Techniques Act, 1994' at http://pndt.gov.in/writereaddata/mainlinkfile/ File100.pdf. Educative material like 'Answers to FAQs on PC-PNDT Act,' a handbook for medical professionals that has been developed by CEHAT for Ministry of Health and Family Welfare with assistance from UNFPA is available at http://pndt.gov.in/writereaddata/mainlinkFile/File55.pdf.

In recent times, a single-point access linking to the most read articles in leading radiology journals has been launched. This has been created primarily to view articles of immediate needs of the academic and practicing radiologist' as well as disseminate the viewing trends in readership. Dynamically, this list of most frequently read articles get updated monthly. The Most Frequently Read Articles can be accessed from the following journal websites: American Journal of Roentgenology at http://www.ajronline.org/reports/ mfr1.dtl, American Journal of Neuroradiology at http://www. ajnr.org/reports/mfr1.dtl, Radiology at http://radiology. rsna.org/reports/most-read and Radiographics at http:// radiographics.rsna.org/reports/most-read. 